\title{
FLUID TRANSPORT MECHANISMS IN MICROFLUIDIC DEVICES
}

\author{
Joshua I. Molho, Amy E. Herr, \\ Thomas W. Kenny, M. Godfrey Mungal \\ Stanford University, Mechanical Engineering Department \\ 551 Terman, Stanford, CA 94305-4021 \\ ph: (650) 725-1595, fx: (650) 723-3521 \\ jmolho@stanford.edu, aeh@stanford.edu
}

\author{
Michael G. Garguilo, Phillip H. Paul \\ Sandia National Laboratories \\ 7011 East Avenue, Livermore, CA 94550 \\ mggarlu@sandia.gov,phpaul@sandia.gov
}

\author{
Manish Deshpande, John R. Gilbert \\ Microcosm Technologies \\ 215 First Street \#219 \\ Cambridge, MA 02142 \\ support@memcad.com
}

\section{ABSTRACT}

Most microfluidic systems rely on one of two manners of fluid transport: pressure-driven or electrokinetically-driven flow. This investigation focuses on describing these flows in microfabricated channels and small diameter capillary tubes. Flow characterization is accomplished by interrogation of micron-scale fluid regions through a powerful, non-intrusive flow imaging technique. Interesting phenomena have been observed from these detailed examinations. Our results are presented in conjunction with an evaluation of mechanisms that potentially explain observed deviations from the HelmholtzSmoluchowski equation. In particular, we show that observed perturbations of electrokinetic flow in open capillaries might be caused by induced pressure gradients. We also show how these induced pressure gradients may globally perturb the flow in an electrokinetically-driven microfluidic system.

\section{NOMENCLATURE}

\begin{tabular}{ll} 
Symbol & \multicolumn{1}{c}{ Definition } \\
$\mathbf{E}$ & electric field \\
$\mathrm{E}_{\mathrm{x}}$ & axial electric field \\
$\mathrm{F}$ & Faraday constant $\left(\mathrm{N}_{\mathrm{a}} \mathrm{e}\right)$ \\
$\mathrm{I}_{0}$ & Modified Bessel function \\
$\mathrm{L}$ & length of capillary \\
$\mathrm{P}$ & Pressure \\
$\mathrm{Q}$ & Volumetric flow rate \\
$\mathrm{R}$ & universal gas constant \\
$\mathrm{S}$ & source term \\
$\mathrm{T}$ & temperature \\
$\mathrm{T}_{0}$ & wall temperature
\end{tabular}

$\begin{array}{ll}\text { Units (SI) } & \mu_{0} \\ \mathrm{~V} \mathrm{~m}^{-1} & \rho_{\mathrm{E}} \\ \mathrm{Vm}^{-1} & \rho \\ \mathrm{C} \mathrm{mol}^{-1} & \zeta \\ & \zeta_{\mathrm{EO}} \\ & \zeta_{\mathrm{EP}}\end{array}$

$\mathrm{m}$

$\mathrm{Pa}$

$\mathrm{m}^{3} \mathrm{~s}^{-1}$

$\mathrm{J} \mathrm{mol}^{-1} \mathrm{~K}^{-1}$

$\mathrm{W} \mathrm{\textrm {m } ^ { - 3 }}$

$\mathrm{K}$

$\mathrm{K}$
$\mathbf{U}$

$\mathbf{U}_{\mathrm{o}}$

$\mathrm{c}_{0}$

$\mathrm{dV}$

$\mathrm{k}$

$\mathrm{r}$

$\mathrm{r}_{0}$

$\mathbf{u}$

$\mathrm{X}$

$\mathrm{x}_{0}$

$\mathrm{y}$

$\mathrm{Z}$

$\varepsilon$

$\phi$

$\lambda_{\mathrm{D}}$

$\mu$

$\mu_{0}$

$\rho_{\mathrm{E}}$

$\rho_{\zeta}$

$\zeta_{\mathrm{EP}}$
Pamela M. St. John, Timothy M. Woudenberg, Charles Connell Perkin-Elmer Applied Biosystems 850 Lincoln Centre Drive, Foster City, CA 94404 StJohnPM@perkin-elmer.com electroosmotic or electrophoretic velocity electrophoretic velocity at $\mathrm{T}_{0}$ ion concentration differential volume thermal conductivity radial dimension capillary inner radius velocity distribution axial dimension axial position of change in wall zeta potential distance from surface charge number permittivity electric potential

Debye length

viscosity viscosity of water at $T_{0}$ electric charge density resistivity zeta potential electroosmotic zeta potential V electrophoretic zeta potential V $\mathrm{m} \mathrm{s}^{-1}$

$\mathrm{m} \mathrm{s}^{-1}$

mol m${ }^{-3}$

$\mathrm{m}^{3}$

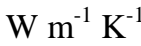

$\mathrm{m}$

$\mathrm{m}$

$\mathrm{m} \mathrm{s}^{-1}$

$\mathrm{m}$

$\mathrm{m}$

$\mathrm{m}$

$\mathrm{C} \mathrm{V}^{-1} \mathrm{~m}^{-1}$ $\mathrm{V}$

$\mathrm{m}$

Pa s

Pa s

$\mathrm{C} \mathrm{m}^{-3}$

ohm m $\mathrm{V}$ V

\section{INTRODUCTION}

Originally emerging from the field of analytical chemistry,


wide interest. Micro total analysis systems and other microfluidic systems rely on both pressure and electrokinetic 
mechanisms for fluid transport. Complicated relationships can arise between the physical characteristics of the microchannels and the behavior of the multi-component fluids flowing through the channels. These relationships have not been systematically studied and are not yet completely understood. In practice, researchers are forced to rely on the use of trial and error in the design of miniaturized fluidic systems.

In this investigation, we employed a caged fluorescence imaging technique in an effort to determine the mechanisms that contribute to the fluid flow in microfluidic systems. Aided with characterizations of relatively simple flows, we endeavor to accurately predict the flow behavior in more complicated flows and geometries.

\section{BACKGROUND}

Electrokinetic transport refers to the combination of electroosmotic and electrophoretic transport. For a historical review of electrokinetic theory, see Burgreen and Nakach (1964). Included below is a brief description of each type of transport.

\section{Electroosmosis}

Electroosmosis refers to the bulk movement of an aqueous solution past a stationary solid surface, due to an externally applied electric field. Electroosmosis requires the existence of a charged double-layer at the solid-liquid interface. This charged double layer results from an attraction between bound surface charges and ions in the passing fluid. In glass capillaries, surface silanol groups become deprotonated and, therefore, are negatively charged. This negatively charged surface attracts positive ions present in the flow. In the situations addressed in this paper, only a very thin layer near the wall has a net charge.

Rice and Whitehead (1965) give a complete analysis of electroosmotic flow in round capillaries; here we quote only a few significant results to which we will refer later. At equilibrium, a Boltzmann distribution of the charges in the solution will exist (Probstein, 1994) with the charge density distribution represented by:

$$
\rho_{E}=z F c_{0}\left[\exp \left(\frac{-z F \phi}{R T}\right)-\exp \left(\frac{z F \phi}{R T}\right)\right]
$$

The potential field is determined by solving the PoissonBoltzmann equation, resulting in the following charge density,

$$
\rho_{E}=\frac{-\varepsilon}{\lambda_{D}^{2}} \zeta_{E O} \frac{\mathrm{I}_{o}\left(r / \lambda_{D}\right)}{\mathrm{I}_{o}\left(r_{o} / \lambda_{D}\right)}
$$

In our experiments, $\lambda_{\mathrm{D}} / \mathrm{r}_{\mathrm{o}}$ is approximately $10^{-4}$; thus the double layer is very thin. The equation of motion for steady, low Reynolds number, incompressible flow is:

$$
\mu \nabla^{2} \mathbf{u}=-\rho_{E} \mathbf{E}+\nabla P
$$

In the limit of a small Debye length, $\lambda_{\mathrm{D}}$, solving Eq. (3) yields the Helmholtz-Smoluchowski equation for the electroosmotic velocity.

$$
U=\frac{-\varepsilon \zeta_{E O} E_{x}}{\mu}
$$

The Helmholtz-Smoluchowski equation predicts a "pluglike" velocity profile when the Debye length is much less than the capillary diameter. The electroosmotic velocity, $\mathrm{U}$, is approximately $0.1 \mathrm{~mm} / \mathrm{sec}$, when $\zeta=0.1 \mathrm{~V}, \mathrm{E}_{\mathrm{x}}=100 \mathrm{~V} / \mathrm{cm}$, and $\mu$ is the viscosity of water (Probstein, 1994).

\section{Electrophoresis}

Electrophoresis describes the motion of a charged surface submerged in a fluid under the action of an applied electric field. Considering the case of a charged dye molecule, it can be shown (Probstein, 1994) that the electrophoretic velocity of the dye is again described by the Helmholtz-Smoluchowski equation.

$$
U=\frac{\varepsilon \zeta_{E P} E_{x}}{\mu}
$$

The electroosmotic zeta potential is a property of the capillary surface while the electrophoretic zeta potential is a property of the charged dye. In general, these two zeta potentials will not be equal.

It is important to note that electroosmosis results in a net mass transfer of the aqueous solution; whereas, electrophoresis causes movement of charged particles or molecules through a stationary solution. For a net-neutral solution of charged molecules, electrophoresis will not result in bulk motion of the solvent. Also note that both derivations shown above result in a velocity that is not a function of the radial position; hence, it is termed a "plug-like" flow.

\section{EXPERIMENTAL DETAILS}

Two different types of microchannels were used in this investigation. Initially, we used rectangular silicone microchannels. We then began investigating flows within round glass capillaries with an internal diameter of $100 \mu \mathrm{m}$. The capillaries were employed in an effort to isolate unexplained flow phenomena observed in our initial silicone microchannel investigation.

To create the silicone channels, silicon microchannel molds were fabricated as "negative" masters using standard 
photolithography. An STS deep reactive ion etch (DRIE) was used to create raised rectangular structures (inverted channels) with dimensions of 10-200 $\mu \mathrm{m}$ in width, $5 \mathrm{~cm}$ in length and 40 $\mu \mathrm{m}$ in height. An RTV silicone (Dow Corning Sylgard 184) was used to form positive replicas of the silicon structures (Effenhauser et al., 1997). A photograph of a sealed replica is shown in Fig. 1. The elastomer channels were sealed with either glass microscope slides or elastomer coated glass microscope slides.

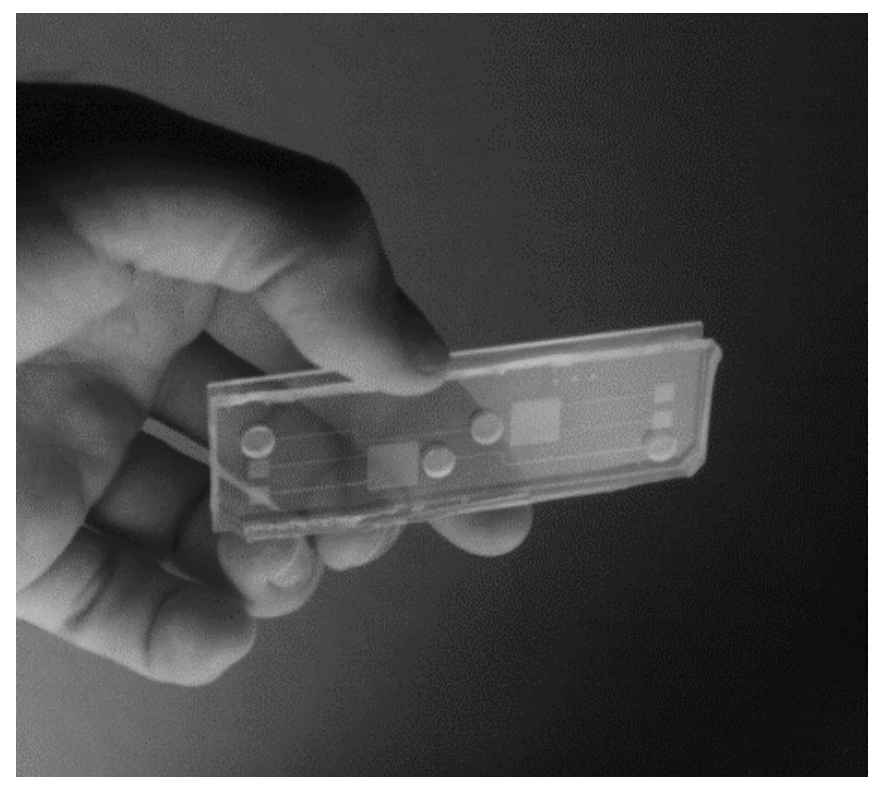

Figure 1. Photograph of the elastomer device with three test microchannels. The circles are the injection wells and the squares are used to study pressure-driven flow in a sudden expansion.

Our experimental approach utilized caged fluorescent dyes to image the flows. These dyes were activated (uncaged) by a UV laser beam and tracked by fluorescence imaging, see Fig. 2, (Paul et al., 1998). In contrast to standard methods injecting dye, this visualization technique allows definition of narrow, fluorescent regions at virtually any location along the channel. Specifically, this allows for a precise definition of a small fluorescent region and therefore an improved determination of the dye profile. Therefore, this caged fluorescence technique has advantages over injection methods that must transport the dye from the location of injection to the region of interest.

The caged dyes were purchased from Molecular Probes Inc. and used in millimolar quantities. For the pressure-driven studies, a caged fluorescein was dissolved in distilled
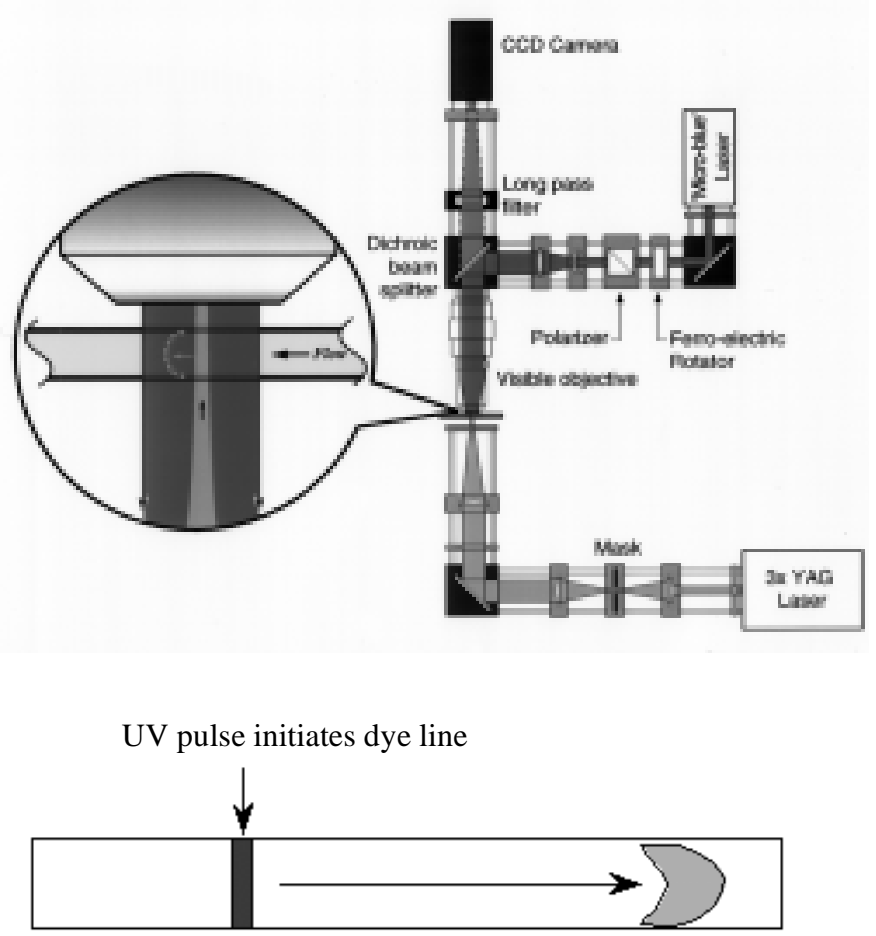

time evolved dye

Figure 2. Top: Schematic of the experiment. The Nd:YAG laser is used to uncage the dye and a Microblue $473 \mathrm{~nm}$ laser is used to excite the fluorescence. Bottom: Illustration of the uncaging process. The uncaging laser defines the starting fluorescent fluid volume.

water. For the electrokinetic studies, a caged rhodamine was dissolved in Tris-EDTA buffer. The dyes are negatively charged when uncaged and may be either charged or uncharged when caged. When caged, the dye solution is non-fluorescent. Upon photo-activation of a UV light illuminated volume, the protecting group is cleaved and the dye becomes fluorescent. Approximately $100 \mu \mathrm{J}$ (per pulse) of $355 \mathrm{~nm}$ light from a pulsed Nd:YAG laser uncaged the dye. The uncaged region extended roughly $20 \mu \mathrm{m}$ along the length of the channel and spanned across its full width (Fig. 2). The laser beam was focused using a UV microscope objective that defined a sharp start zone. A continuous wave Microblue diode pumped laser at $473 \mathrm{~nm}$ (Uniphase) was used for fluorescence excitation after activation (Fig. 2). Fluorescence images of the molecules diffusing and moving in the local flow were collected using a microscope objective and a video-rate, interlaced camera (Texas Instruments MC-780PH). 


\section{RESULTS}

We have investigated transport in microfluidic systems and present experimental studies of pressure-driven and electrokinetic flows. We also present numerical simulations which were performed using the NetFlow module in Memcad (MEMCAD, 1998). The NetFlow module employs a threedimensional finite element based tool to solve the NavierStokes equations. We note that the pressure-driven flow data show good agreement between the preliminary experiments and the simulations. Measurements of the electrokinetic flows, however, reveal deviations from simple theory.

The evolution of a pressure-driven fluorescent profile is compared to a corresponding sequence of simulations in Fig. 3. The agreement is good, but note that there is dye retention near the walls that is not duplicated in the numerical simulations. This dye retention could be due to surface roughness. Atomic force microscopy indicates that the silicone channel has an RMS surface roughness of $\sim 7 \mathrm{~nm}$.

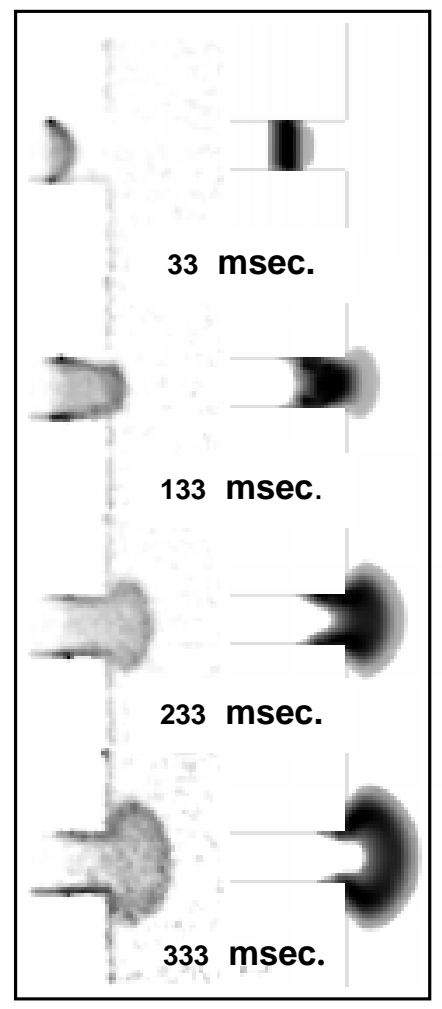

Figure 3. Experimental (left) and simulated (right) pressuredriven flow entering a sudden expansion in an elastomer structure. The channel entering the expansion is rectangular (100 $\mu \mathrm{m}$ wide and $40 \mu \mathrm{m}$ deep)

The evolution of an electrokinetically-driven fluorescent profile in a round, fused-silica capillary is shown in Fig. 4. These images reveal that the velocity profile appears to be constant across the channel, in agreement with Eq. (4). Figure 5 shows electrokinetic flow in a DBwax-coated (polyethylene

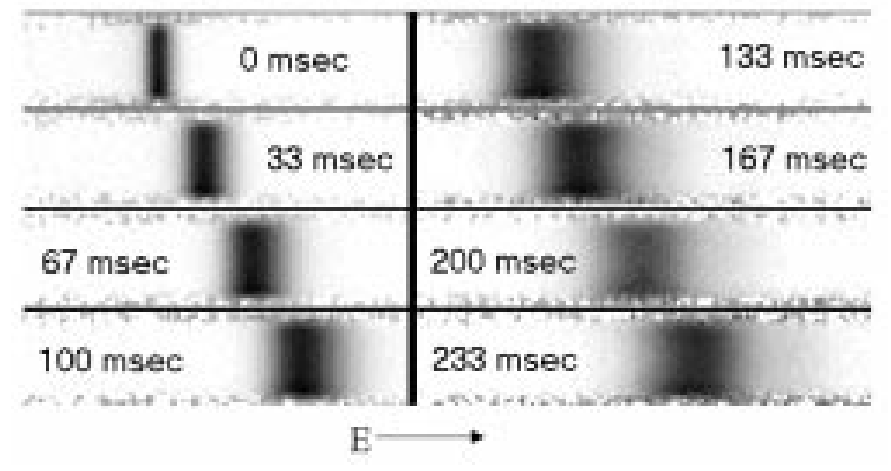

Figure 4. Electrokinetic flow in a $75 \mu \mathrm{m}$ diameter fusedsilica capillary exhibiting a plug-like velocity profile. Applied field strength was 350 volts $/ \mathrm{cm}$. The numbers with each image correspond to the time in milliseconds after the uncaging pulse.



Figure 5. Electrokinetic flow in DBwax-coated (polyethylene glycol), $100 \mu \mathrm{m}$ diameter fused-silica capillary. The waxcoated capillaries were purchased from J\&W Scientific. Applied field strength was 245 volts/cm. The numbers with each image correspond to the time in milliseconds after the uncaging pulse.

glycol) capillary. The wax coating is used to suppress electroosmotic flow, so that the movement of charged particles in the flow is due to electrophoresis only. However, these images show a pronounced parabolic velocity profile in the direction opposing the electric field. This profile does not agree with Eq. (5), and would be difficult to image without a high-resolution measurement technique such as caged florescence imaging. Note that we observed no motion of the dye without application of the electric field, verifying that there was no externally applied pressure gradient. Parabolic components to the electrokinetic velocity profile have previously been observed in capillaries (Paul et al., 1998) and in microchannels (St. John et al., 1998). 


\section{DISCUSSION}

In Fig. 4, we see that the imaged velocity profiles for electrokinetic flow are in good agreement with Eq. (4). The flow is a combination of electroosmosis and electrophoresis, and the theory outlined above shows that both components yield a flat profile. Figure 5, however, shows significant deviations from a flat profile. This flow suggests a velocity profile similar to that observed in a pressure-driven flow.

In the following sections, we explore two mechanisms that potentially explain this observed departure from plug-like flow: variations in viscosity due to temperature gradients, and induced pressure gradients caused by a non-uniform wall potential (i.e. non-uniform wax coating in the case of Fig. 5).

\section{Variation of viscosity}

In this section, we examine the possibility that heat generation within the capillary could cause the electrophoretic velocity profile to deviate from plug-like. To this end, we investigate the temperature, viscosity and corresponding velocity distributions across the capillary.

In cylindrical coordinates, we write the temperature governing equation for our system as:

$$
-\frac{1}{r} \frac{d}{d r}\left(k r \frac{d T}{d r}\right)=S=\frac{E_{x}^{2}}{\rho} .
$$

The source term, S, has units of power per unit volume. In this analysis, we assume $\rho$ remains a constant (or at least is not a strong function of temperature) based on the experimentally determined fluid-filled capillary resistance and capillary dimensions. This empirically determined resistivity agrees closely with those tabulated in the literature (Masliyah 1994). Integrating twice and applying the boundary conditions of finite temperature at the centerline and constant wall temperature, $\mathrm{T}_{\mathrm{o}}$, we obtain the temperature distribution

$$
T(r)=\frac{E_{x}^{2} r_{o}^{2}}{\rho 4 k}\left(1-\frac{r^{2}}{r_{o}^{2}}\right)+T_{o} .
$$

As an initial approximation, we assume that the fluid within our capillary has the same thermal properties and viscosity as water. As such, we have chosen to model the viscosity as a function of temperature, by:

$$
\frac{\mu(T)}{\mu_{o}}=\exp \left[a+b\left(\frac{T_{o}}{T}\right)+c\left(\frac{T_{o}}{T}\right)^{2}\right]
$$

with $\mathrm{T}_{\mathrm{o}}=293.15 \mathrm{~K}$ and the constants given by a $=-1.94$, $\mathrm{b}=-4.80$, and $\mathrm{c}=6.74$, as suggested by Reid (1987).

In order to relate the temperature-dependent viscosity to the electrophoretic velocity profile within the capillary, we return to our analysis of electrophoresis. Utilizing Eq. (5) and neglecting the temperature dependence of $\varepsilon, \zeta$, or $E_{x}$, we arrive at:

$$
U(T)=\frac{\varepsilon \zeta_{E P} E_{x}}{\mu(T)} .
$$

The predicted velocity profiles for both an open capillary and a DBwax-coated capillary each show an expected radial velocity variation of less than $0.2 \%$ (Fig. 6). The radial velocity distribution is parabolic in nature, but its magnitude is too small to explain the dye profiles seen in Fig. 5. To obtain a significant radial variation in the electrophoretic velocity, one would need to increase the field strength or the buffer concentration considerably. Our temperature variation model and, hence these conclusions, are in close agreement with those presented by Grushka et al. (1989).

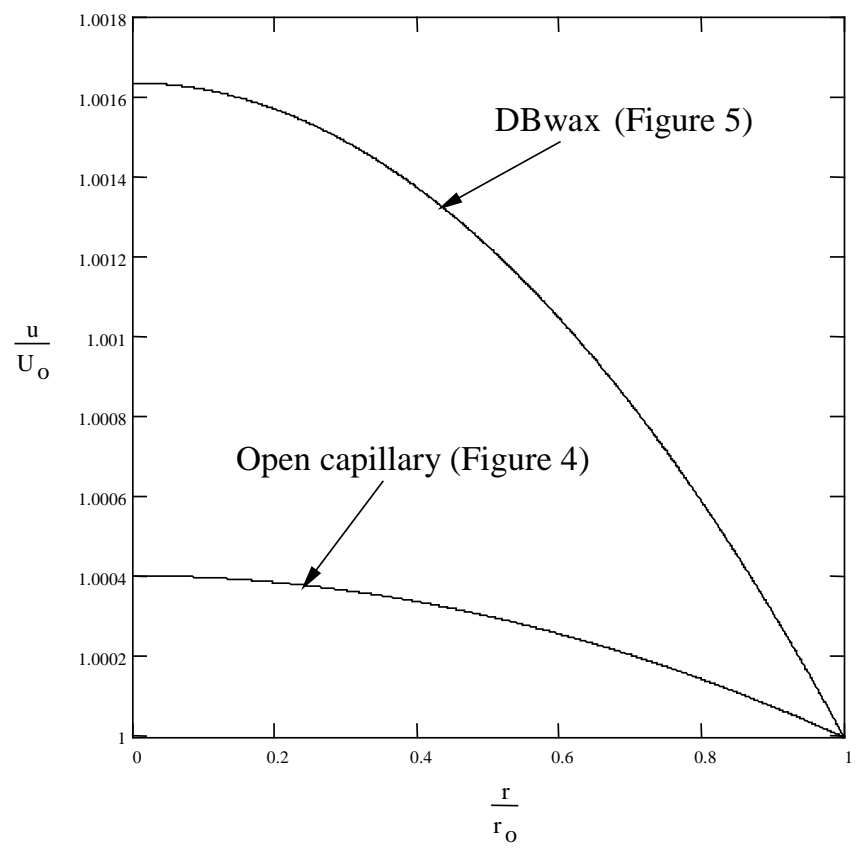

Figure 6. Electrophoretic velocity distribution for an open capillary and a DBwax-coated capillary

From this analysis, we conclude that the viscosity and, therefore, velocity do not vary significantly across the capillary for the prescribed small-bore capillary parameters, buffer concentrations, and field strengths. Therefore, Joule heating induced velocity variation does not appear to be a feasible mechanism for the observed deviations from plug-like flow.

\section{Induced Pressure Gradients}

In deriving Eq.(4), the pressure gradient term was neglected because there were no externally applied pressure gradients. However, this expression will not be correct if the electrokinetic transport induces a pressure gradient. We now explore how the electrokinetic flow could potentially induce 
pressure gradients. If we take the divergence of Eq. (3), and apply continuity for the fluid, $(\nabla \cdot \mathbf{u})=0$, we derive the following expression.

$$
\nabla^{2} P=\mathbf{E} \cdot \nabla \rho_{E}+(\nabla \cdot \mathbf{E}) \rho_{E}
$$

Equation (10) demonstrates how the pressure field evolves from gradients in the charge density and applied field. Referring to Eq. (2), we see that the first term on the right hand side of Eq. (10) will be non-zero if the wall potential changes in the axial direction. Nonuniformities in the wall potential could exist due to contamination in the capillary, variations in wall coatings, or gradients in the buffer $\mathrm{pH}$. Using classical techniques for solving Poisson's equation (e.g. Green's functions), it is possible to develop a general solution to Eq. (10) for an arbitrary zeta potential distribution along the wall of a cylindrical capillary. In this paper, a direct method will be used to solve for the pressure field in a capillary with a wall potential as shown in Fig. 7.

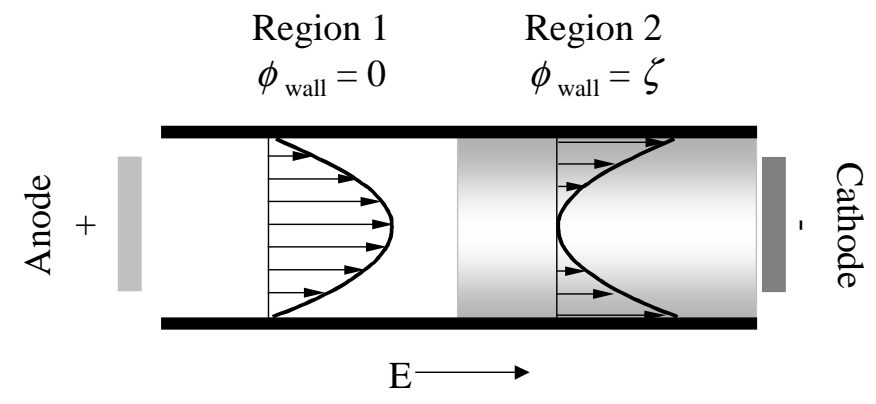

Figure 7. Schematic of capillary with step change in wall potential. The anticipated velocity profiles are also shown.

Let us assume that the fluid velocity is only a function of radial position in both Region 1 and Region 2 shown in Fig. 7. Note that Region 1 describes a section of the capillary with zero wall potential; whereas, Region 2 has a finite wall potential. In other words, we will assume that axial velocity gradients are confined to a vanishingly small region near the discontinuity in wall potential. With this assumption, the volumetric flow rates in regions one and two are shown below.

$$
\begin{aligned}
Q_{1} & =\left(-\frac{\partial P}{\partial x}\right) \frac{\pi r_{o}{ }^{4}}{8 \mu} \\
Q_{2} & =\left(-\frac{\partial P}{\partial x}\right) \frac{\pi r_{o}^{4}}{8 \mu}+\frac{\varepsilon \zeta E_{x}}{\mu} \pi r_{o}^{2}
\end{aligned}
$$

We consider the case where the pressure at either end of the capillary is a known reference value (i.e. atmospheric pressure). For an incompressible liquid, the volumetric flow rates must be the same in regions 1 and 2. Furthermore, we know that the pressure is continuous and piecewise linear since we assumed that the velocity profile does not vary axially in either region (i.e. the pressure gradient must be constant in both regions). Therefore, we arrive at the following expression for the pressure in the capillary, where $\mathrm{x}$ is the axial position and $\mathrm{x}_{\mathrm{o}}$ is the axial location of the discontinuity in wall potential.

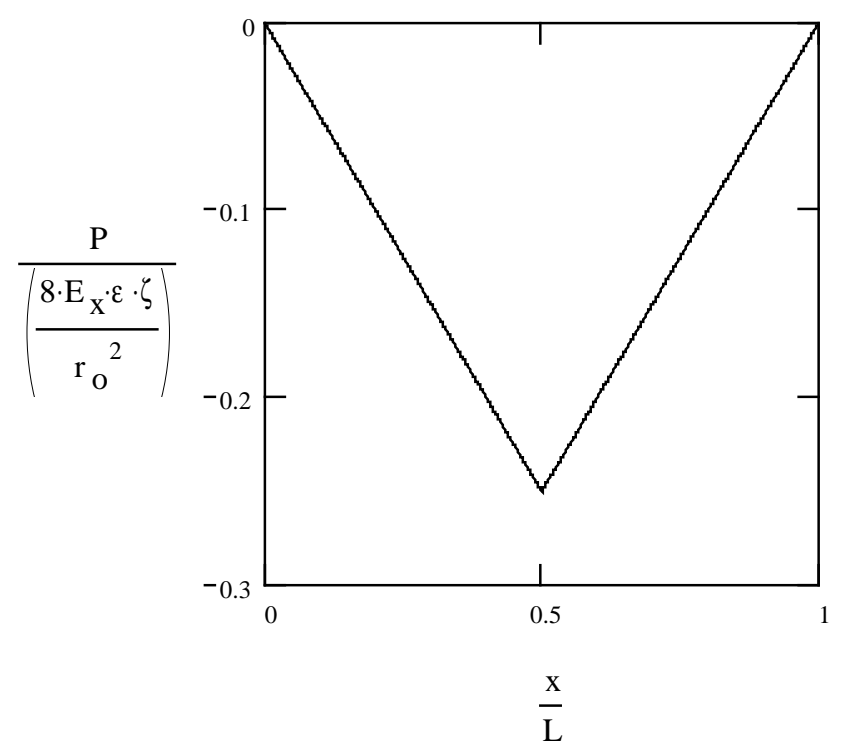

Figure 8. Non-dimensional pressure in a capillary with a step change in wall potential.

$$
P(x)=\frac{8 E_{x} \varepsilon \zeta}{r_{o}^{2}}\left\{\begin{array}{l}
-\frac{x}{L}\left(L-x_{o}\right), x<x_{o} \\
-\frac{x_{o}}{L}(L-x), x \geq x_{o}
\end{array}\right.
$$

The pressure field corresponding to Fig. 7 is plotted in Fig. 8. We see that the pressure gradient is constant in each section and acts to ensure that the mass flow rates are equal in each region. Figure 9 shows the predicted velocity profiles in regions 1 and 2 . The velocity in the first section is due to pressure alone, since the wall potential there is zero. The velocity profile in the second section is simply a superposition of the flat electroosmotic profile and the Poiseuille profile due to the induced pressure gradient. The pressure gradient in each region is readily calculated from Eq. (12). The velocity profiles in Fig. 9 were also shown schematically in Fig. 7. 


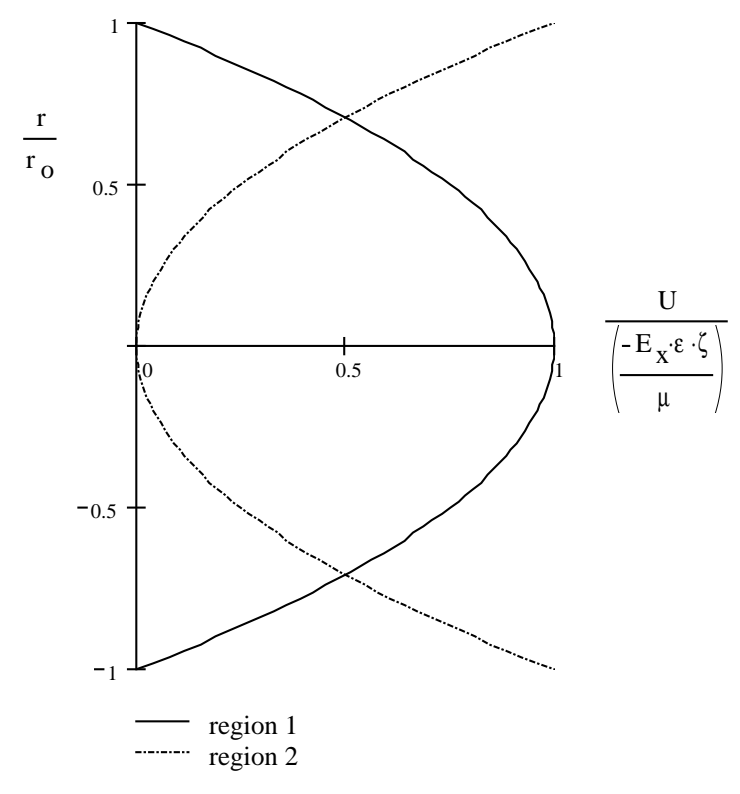

Figure 9. Radial velocity profiles in region 1 and region 2.

To understand the solution qualitatively, one can imagine that the electroosmotic flow in Region 2 "pulls" the flow in Region 1. In other words, the electroosmotic movement of liquid in Region 2 tends to create suction at the interface between the two regions. Hence, the pressure drops at the interface until the mass flow rates in each region are equal. Note that the pressure-driven component subtracts from the electroosmotic flow in Region 2 while only pressure-driven flow is present in Region 1. The analytically calculated velocity profiles also agree qualitatively with the numerical simulation shown in Fig. 10 (MEMCAD, 1998). The simulation confirms that axial gradients in the velocity field are confined to a region less than a single diameter from the wall potential discontinuity.

Superpositions of Eq. (12) can be used to find the pressure field caused by one or many small regions of zero wall potential. This is then a model of a "dirty" silica capillary. Figure 11 demonstrates the nondimensional pressure field in a capillary with three "dirty" regions, each covering $1 \%$ of the capillary's length.

Note that the pressure gradients in the clean regions are identical and that the localized discontinuities in wall potential perturb the flow everywhere. Equation (12) can also be superposed to find the pressure field in a coated capillary (zero wall potential) with small "clean" regions where the wall

$$
\begin{array}{cc}
\text { Region 1 } & \text { Region 2 } \\
\phi_{\text {wall }}=0 & \phi_{\text {wall }}=\zeta
\end{array}
$$

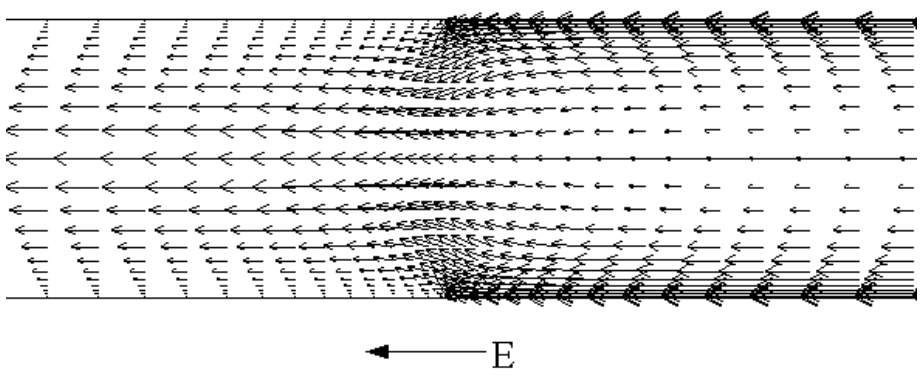

Figure 10. Simulation of flow in a capillary with a step change in wall potential.

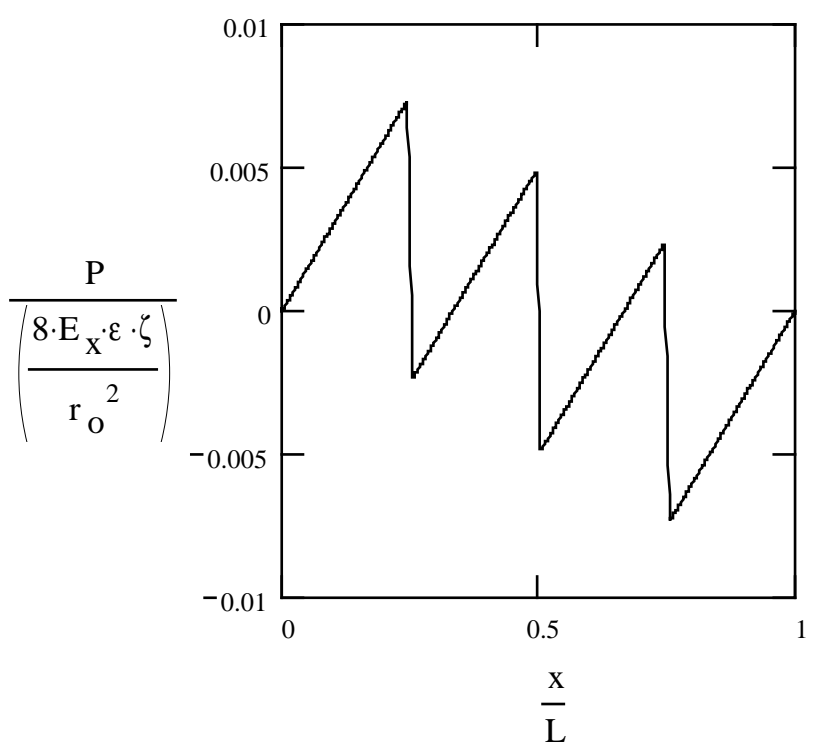

Figure 11. Nondimensional pressure field in a capillary with three "dirty" spots

potential is non-zero. Figure 12 shows the resulting velocity profile in the coated regions, for various amounts of exposed (uncoated) capillary. The induced pressure gradient model presents a rational explanation of the flow imaged in Fig. 5, as it admits a parabolic profile for reasonable variations in wall potential. 


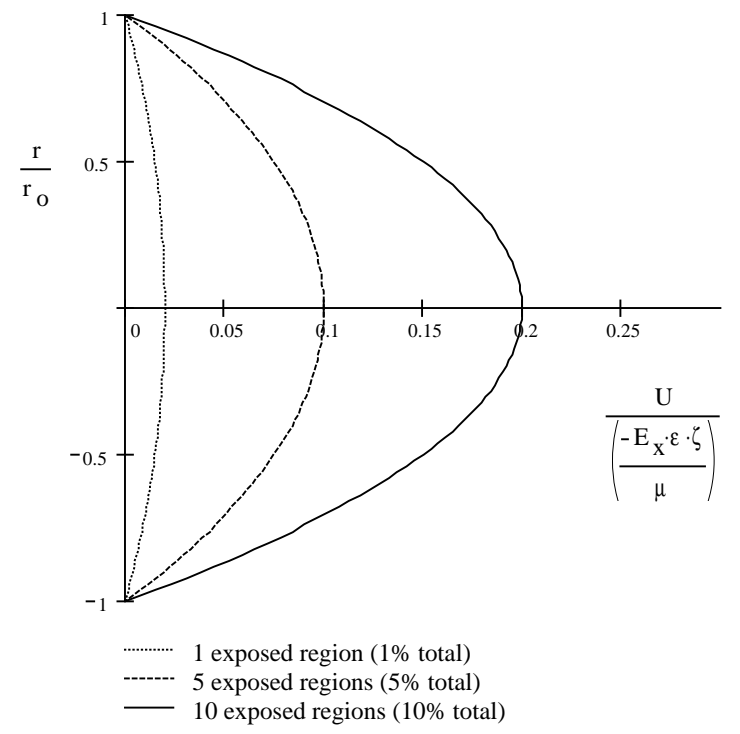

Figure 12. Velocity profile in a coated region of a wax coated capillary with small regions uncoated. The wax coating acts to prevent electroosmotic flow.

\section{CONCLUSIONS}

We have imaged pressure-driven and electrokineticallydriven flows in silicone microchannels and silica capillaries using a powerful caged fluorescence technique. The pressuredriven flows agree with theory and numerical simulations. Simple theory predicts that the electrokinetic flows should display uniform (plug-like) velocity profiles. However, in some cases, we observe large parabolic-like components to the electrokinetic velocity profiles.

We explored two mechanisms in an attempt to explain the observed velocity profiles: viscosity variations and induced pressure gradients. We have demonstrated that Joule heating and the subsequent temperature and viscosity gradients do not adequately explain the observed parabolic velocity profile, but that induced pressure gradients are a possible explanation. An important consequence of the induced pressure gradient analysis is that small regions of non-uniform wall potential create pressure gradients everywhere in the flow. This conclusion has important implications for those wishing to build lab-on-a-chip devices, since pressure gradients tend to increase the dispersion of sample packets in the flow. We are currently performing experiments that investigate the proposed induced pressure gradient mechanism.

\section{ACKNOWLEDGEMENTS}

This work was funded in part by DARPA Composite CAD (Grant no. F30602-96-0306), the National Science Foundation, and Stanford University. The authors would also like to acknowledge assistance from Stanford Professors Juan Santiago and Joel Ferziger.

\section{REFERENCES}

1. Burgreen, D., Nakache, F.R., 1964, "Electrokinetic Flow in Ultrafine Capillary Slits." The Journal of Physical Chemistry, Vol. 68, No. 5, pp. 1084-1091

2. Effenhauser, C. S.,. Bruin, G. J. M, Paulus, A., and Ehrat, M., 1997, "Integrated capillary electrophoresis on flexible silicone microdevices: Analysis of DNA restriction fragments and detection of single DNA molecules on microchips," Anal. Chem., Vol. 69, p. 3451.

3. Grushka, E., McCormick, R. M., and Kirkland, J. J., 1989, "Effects of temperature gradients on the efficiency of capillary zone electrophoresis separations," Anal. Chem. Vol. 61, p. 241

4. Mala, G. M., Li, D., Dale, J. D., 1996, "Heat transfer and fluid flow in microchannels," American Society for Mechanical Engineers, Vol. 59, p.127

5. Manz, A., Effenhauser, C. S., Burggraf, N., Harrison, D. J., Seller, K., and Flurl, K., 1994, "Electroosmotic pumping and electrophoretic separations for miniaturized chemical analysis systems", J. Micromech. Microeng., Vol. 4, p. 257

6. Masliyah, J. H. 1994. Electrokinetic Transport Phenomena, Aostra Technical Publications, Alberta.

7. MEMCAD v4.0, Microcosm Technologies, Inc. (www.memcad.com) (1998).

8. Paul, P.H., Garguilo, M.G. and Rakestraw, D.J., 1998 "Imaging of Pressure- and Electrokinetically-Driven Flows Through Open Capillaries," Anal. Chem., Vol. 70, pp. 2459-2467.

9. Probstein, R.F., 1994 Physiochemical Hydrodynamics: An Introduction. $2^{\text {nd }}$ ed., Wiley and Sons, Inc., New York.

10. Qui, X. C., Hu, L., Masliyah, J. H., and Harrison, D. J., 1997, "Understanding fluid mechanics within electrokinetically pumped microfluidic chips", 1997 International Conference on Solid-State Sensors and Actuators, Chicago, IL.

11. Rice, C.L., Whitehead, R., 1965, "Electokinetic Flow in a Narrow Cylindrical Capillary," The Journal of Physical Chemistry, Vol. 69, No. 11, pp. 4017-4023.

12. Reid, R.C., Prausnitz, J.M., and Sherwood, T.K., 1987, The Properties of Gases and Liquids, $4^{\text {th }}$ ed., McGraw-Hill, New York.

13. St. John, P. M., Deshpande, M., Molho J. I., Garguilo, M. G., et al., 1998, "Metrology and Simulation of Chemical Transport in Microchannels," 1998 Solid-State Sensor and Actuator Workshop, Hilton Head Island, SC., pp. 106-111. 